\title{
Hull Structures as a System: Supporting Lifecycle Analysis
}

\author{
Matthew Collette
}

\begin{abstract}
Hbstract
As modular weapon systems allow cost-effective upgrades of a vessel's war-fighting capability, the degradation of the difficult-to-upgrade structure of the vessel may soon become one of the key drivers of vessel retirement and lifecycle maintenance costing. Existing structural design approaches are reviewed, along with recent developments in this field. It is argued that recent research has produced a number of ad hoc metrics for structural design, such as producability; however, to truly address the needs of future ship design teams it is necessary to integrate several such metrics in a systems-engineering view to evaluate how the structural system contributes to the overall capabilities and costs of a proposed vessel. Potential architectures for this approach are discussed, along with key shortcomings. A comparative example is given for structural fatigue of a strength deck under global bending loading, comparing the traditional design approach with a systems-oriented view.
\end{abstract}

\section{Introduction}

The US Navy is currently faced with the challenge of approving both innovative structural designs, such as the all-aluminum trimaran LCS, and maintaining the structure of existing conventional vessels, such as the CG-49 and DDG-51 classes. Despite extensive research into structural analysis and structural healthmonitoring technologies, naval architects currently lack the tools to forecast lifecycle structural performance and maintenance costs during either design or through-life extension studies. Such lifecycle considerations are expected to grow in importance as modular weapon and combat information systems allow cost-effective through-life upgrades, removing weapon system obsolescence as a reason to retire vessels from service. Furthermore, for naval structures, the structural system is typically supporting an investment of weapons, sensors, machinery, and other vessel systems worth many times the value of the structure itself but effectively permanently tied to the structure. In the future, it is highly likely that degradation of difficult-to-upgrade hull structure will be one of the primary driving causes for retiring a vessel from service, and thus directly impacting the overall platform lifecycle cost (LCC). Thus, the ability to predict and control structural lifecycle and maintenance costs will become increasingly important as the Navy considers service life extensions or evaluates suggestions for radical departures from conventional designs, such as the proposed 100-year service life ship.

To effectively support future ship design, maintenance, and service-life extension decisions, it is proposed that it is necessary to extend the existing semiempirical component-based structural design rules based primarily on safety concerns to a system performance model for ship structures. This system-based approach extends the existing rule-based approach by formally stating performance requirements for the structure 
based on the vessel systems that the structure supports, and considering maintenance and repair requirements during structural design and analysis. By explicitly setting such performance targets, it will be possible to assign key performance parameters (KPP) and key system attributes (KSA) to the structural system, and allow the overall vessel design synthesis work to evaluate different structural concepts and weight budgets against achieved vessel performance attributes. This would allow the impact of designing for increased structural performance (such as extended lifetime or a reduction in the estimated repairs) to be traded off against impacts on other aspects of design such as displacement or powering requirements. Beyond design, these formal system targets would allow real-world experience with the vessel to be interpreted as an ongoing validation trial of the structural system, and observed failures, maintenance actions, and potential service-life extension options can be compared in terms of the system performance they provided for the time and monetary resources that they consume.

However, moving to such a model is complex, as many performance requirements are built into the existing structural semiempirical rule sets without being formally identified, making it difficult to develop a complete set of requirements for new structural designs. This is especially true if service life is to be treated as a design variable, many rule sets implicitly assume a 20-30-year service life and cannot evaluate the impact of stretching the service life to 40,50 , or 100 years. Likewise, existing structural maintenance activities and costs are rarely fed back to naval ship designers, and thus it is rare to formally consider the impact on maintenance downtime or cost when reviewing different structural design options. In this paper, existing structural design, production, and maintenance philosophy for military and commercial vessel structures are reviewed. Achieved service lives under the existing approaches are also documented. Based on the existing state-of-the-art, developments required for a system-based model of structural performance are discussed.
By adapting elements of system engineering to the structural design problem, an initial architecture for a structural system performance model is presented. Conclusions on the applicability of the systems view to structural design and analysis are presented, along with recommendations for future technology development to support the systems modeling approach.

\section{Current Practice NAVAL VESSELS}

The structural design of naval vessels is a complex undertaking, as the magnitude of independent loads such as slamming pressures are difficult to estimate even in probabilistic terms, as are the combined effect of multiple loads acting simultaneously. Additionally, the true structural strength is also difficult to predict, as stiffened shell structures such as ships typically fail through complex buckling behavior involving geometric and material nonlinearities. Against this background, existing naval vessels are often designed by experiencecalibrated design processes, such as those captured in US Navy Design Data Sheets or more recently, the American Bureau of Shipping Naval Vessel Rules. Sielski (2007) presents an overview of the philosophy behind this procedure, which is shown in Figure 1. In Figure 1 characteristic loads are used along with established analysis procedures and acceptance criteria to develop the design of a naval structure. The acceptance criteria is noted as "allowable stress," which is often encountered, but the same design philosophy applies when other criteria are used for acceptance, such as predicted ultimate hull girder strength under UNDEX loading by tools such as ULTSTR. When consistent analysis procedures and load determination is used, acceptance criteria can be developed by applying the load and analysis methods to existing successful vessels. Costing is not explicitly considered in this structural design approach, historically weight-based costing approaches have been used to estimate initial build cost. The shortcomings of weight-based approaches have been well documented, and more recently improved PODAC (Barentine 2010) build cost 
models have been proposed. Maintenance costs have typically not been considered at the design stage. Owing to the size and cost of the structure, full-scale prototypes are not built and tested to validate achieved performance of the structure, although subcomponents may be tested for fatigue or ultimate strength.

The existing design philosophy has worked well for ships where structural life and maintenance costs were not key design drivers. However, as the approach uses characteristic loads and acceptance criteria that do not correspond to actual failure points or reduction in mission capability, it is difficult to directly extend the current approach to support lifecycle assessment. This is especially true for midlife assessments, where measured loads and reports of actual damage may be available, but with no clear path of how to use them in the framework shown in Figure 1. The average achieved service lives of US Navy vessels under the existing design approach are summarized in Table $\mathbf{1}$, based on commissioning and decommissioning dates. Note that structural condition is often not the primary reason for retiring these vessels. However, it is interesting to note that the achieved service life tracks roughly with platform cost, and the only classes achieving average lives in excess of 30 years are those that did not serve continuously or underwent extensive midlife retrofits, such as the Service Life Extension Program for the aircraft carriers.

Naval vessel structural design has continuously evolved, with recent developments including the increasing prevalence of naval rule sets at major classification societies, which outsources part of the rule development and compliance verification from the naval administration to an external body. Paralleling developments in the commercial marine industry, NATO has recently developed ANEP 77 (NATO 2010), a code for naval ship certification using a goal-based standards (GBS) approach. This aims to correct one of the current deficiencies in empirically based standards, namely that the intent of each regulation in the standard is often opaque, making

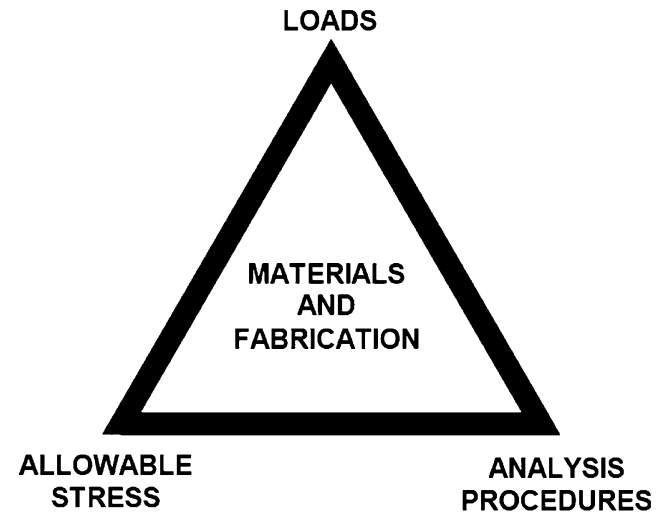

Figure 1: Current Structural Design Procedure after Sielski (2007)

assessment of equivalence for novel arrangement, new materials, or cost-savings reasons difficult. ANEP 77 uses a pyramid approach as shown in Figure 2, defining aims, goals, functional areas, performance requirements, verification methods, and justification for naval vessels. In this approach, the code has a single aim, namely to protect life onboard naval vessels to a standard not less than that on merchant vessels. Each major section of the code has a goal, which is then subdivided into functional areas, specific performance requirements, and

\section{TABLE 1: Average Achieved Service Life}

\begin{tabular}{lc} 
Ship Type & Average Service Life Years \\
\hline Carriers $^{a}$ & 40.4 \\
\hline BB-6l Class $^{b}$ & 48.8 \\
\hline Amphibious Vessels $^{c}$ & 29.2 \\
\hline Cruisers $^{d}$ & 26.3 \\
Destroyers $^{\text {e }}$ & 25.4 \\
Frigates $^{f}$ & 19.8
\end{tabular}

ancludes data from Forrestal and Kitty Hawk classes, John F. Kennedy, and planned decommissioning of Enterprise in 2013.

${ }^{\mathrm{b}}$ Includes extensive time in reserve.

cIncludes 60 amphibious assault and carogo ships from the cold war era retired through 2009.

${ }^{d}$ Data from Koenig et al. (2009) includes 32 cruisers nuclear and non-nuclear cruisers from cold war era up to decommissioned CG-47 hulls.

eData from Koenigg et al. (2009) includes 91 destroyers from cold war era through decommissioned DD-963 hulls. 'Data from Koenig et al. (2009) includes 103 frigates from cold war era through decommissioned FFG-7 hulls. 
then either prescriptive or performance-based verification methods are specified to assess if a ship complies with the code. Finally, justification is given for future maintenance and management of the code. The code covers structures, hydrostatics, engineering systems, fire safety, as well as other areas of vessel design. ANEP 77 does include some discussion of ongoing inspection and surveys throughout the vessel's life to ensure continued compliance with the code, however, it is not the intent of these sections to formally consider the lifecycle impact of alternative structural configurations, except as they relate to safety inspections.

The ANEP 77 approach is fundamentally about life safety. As noted in the "Introduction" and "Current practice" of ANEP 77, the ANEP 77 code is inspired by International Maritime Organization (IMO) conventions and resolutions whose primary focus is safety of life, environment, and property.

Work on approaches with explicit performance metrics for naval structures has been very limited to date. The work that has been done is primarily focused on reducing build cost through improved producability of the structure. Hess (2002) proposed one of the few naval approaches for in-service performance metrics for structural designs, using structural reliability calculations as a way of defining durability, dependability, and capability metrics for the structure's performance throughout its lifecycle. This approach adapted the framework for nonstructural systems proposed in previous editions of OPNAVINST 3000.12 (OPNAV 1987), although the framework used by Hess has not been extended to the current edition of the instruction.

\section{COMMERCIAL VESSELS}

The approach to commercial vessel structural design is similar in many respects to the approach to naval vessel structural design. Classification society rule sets are used throughout the industry to set a minimum standard for vessel structures, and ongoing structural safety is assured through periodic inspections and surveys carried out by classification societies on behalf of the owners. Owners are free to design structures beyond the minimum standard required by classification societies, and some operators frequently do so, if they feel that the reduced maintenance requirements or longer asset lifetime will give them an economic advantage in the marketplace.

Similar to ANEP 77, GBS have been a topic of great debate over the last two decades, with

Figure 2: NATO ANEP-77 Approach after NATO (2010)

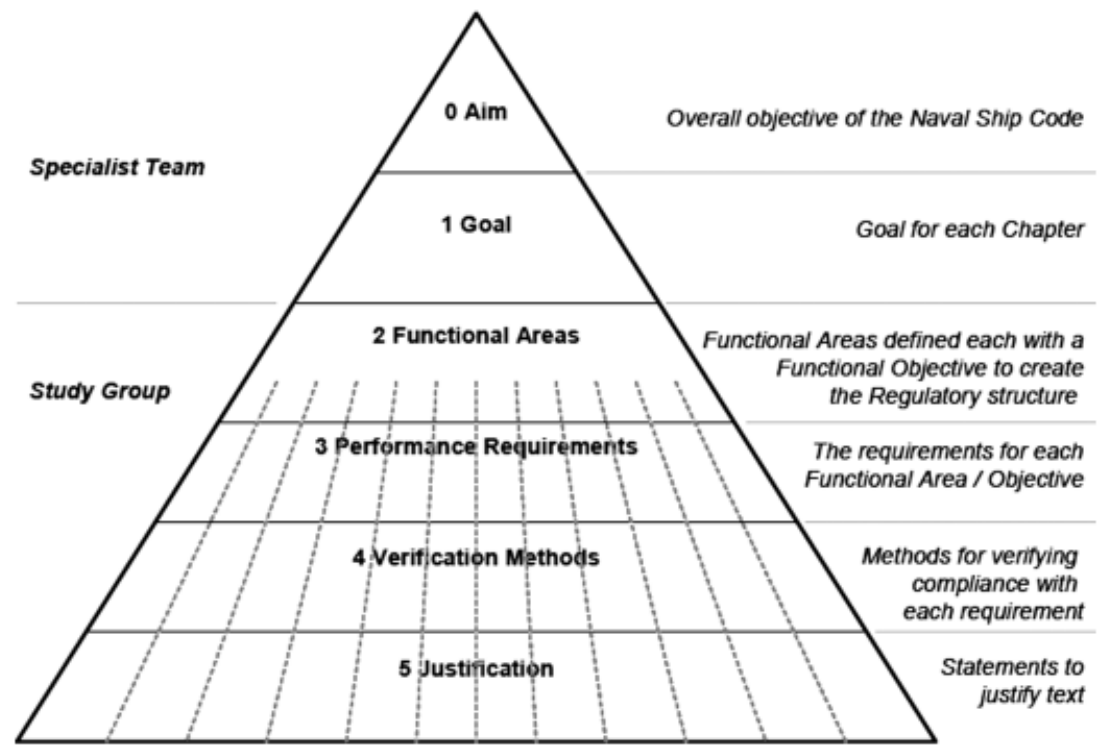


IMO formally adopting a goal-based approach (Hoppe 2005) to ship standards, with the initial application to tankers and bulk carriers over $150 \mathrm{~m}$ in length coming into force on May 10, 2010. Similar to the ANEP 77 code, the commercial GBS follow a pyramid-like approach, with five different tiers. The IMO has primarily been involved in the upper two tiers, setting overall goals and some functional requirements, such as that the minimum design life for new oil tankers and bulk carriers shall be at least 25 years, and all ships shall be designed to operate in realistic environmental conditions such as the North Atlantic. Verification in the IMO GBS approach involves verifying that the rule sets used in tiers four and five achieve the goals of tiers one and two. Verification of rule sets is performed on a self-verification basis by the classification societies, who are also responsible for assessing individual ship's compliance with these rule sets.

The development of GBS has occurred in parallel with the common structural rules that have been developed for tankers and bulk carriers by various members of the International Association of Classification Societies. Implementing many aspects of GBS, these rules include new performance-based requirements, such as explicit corrosion allowances which allow the owner to add additional material above that required by the rules if they feel that the reduction in plate renewal throughout the vessel's life resulting from the additional allowance is economically competitive.

Efforts to go beyond rule-based approaches to structures and replace them with explicit performance metrics have been proposed in the commercial world as well. Work on design-forsafety, largely spearhead by the efforts of the European research community has resulted in proposed approaches for safety metrics for highspeed craft structures (Collette et al. 2005), and the integration of structural design into more general design-for-safety approaches (Sames and Hamann 2008). Given the tough economic environment of commercial shipyards, productionbased metrics for structural design have also been extensively developed to decrease ship construction costs (Rigo 2001). More recently, these approaches have been extended to consider the entire ship lifecycle, balancing production costs against in-service downtime for repairs and related through-life expenses (Turan et al. 2009).

\section{SUMMARY OF EXISTING APPROACHES}

In the marine community, established structural design procedures mainly revolve around the design approach shown in Figure 1. While recently complex analysis approaches such as finite-element analysis have replaced simple analysis methods, the overall philosophy has remained largely the same. The move toward GBS has clarified the intent behind the design rules used to implement the design approach in Figure 1. This clarification makes determining equivalence easier for novel design, but again, does not expand the fundamental design approach, which remains focused on ensuring structural safety under operational conditions. Recent naval-specific GBS such as NATO ANEP 77 have moved GBS developments from the commercial world into the military world, but have not extended the focus of the structural design methods beyond ensuring structural safety. Metric-based design approaches for structures have also been proposed in the marine industry, again, primarily dealing with safety or designfor-production issues, with relatively few examples dealing with lifecycle costing or performance issues such as dependability and durability. Almost all approaches developed so far in this regard have focused on the design of new structures, and the use of metric-based approaches has primarily been on an ad hoc basis.

\section{The Way Hhead—H Systems View?}

At the current time, naval structural design is primarily based on achieving compliance with codes such as Naval Vessel Rules, with ad hoc application of metric-based approaches for issues such as producability of the resulting structure. Given the importance of the structure in the overall lifecycle of a high-value Navy warship, it is proposed that a wider view of the structural design problem would be beneficial. 
While compliance with codes such as the Naval Vessel Rules ensures a minimum level of structural safety, many different structures could be synthesized that meet the requirements of the Naval Vessel Rules, yet have vastly different LCC and capabilities. At the current time, there is no formal method for the overall acquisition program to inform the structural design team of the relative importance of different LCC and capabilities when deciding which structure to settle on. To address this need, it is proposed to extend the metric-based approaches to structural design in a more formal way, namely using high-level systems engineering techniques to define key requirements for the structural system, and then to compute explicit metrics to verify that candidate structural designs meet these requirements. This would in effect join together the metrics that have been proposed for production and safety with new metrics for capability and other design-specific requirements in a logical fashion based on the needs and priorities of the overall design effort. Such an approach can also be extended to lifecycle maintenance, where specific maintenance actions can be reviewed to determine the most effective method of guaranteeing a specific level of structural performance in operation.

System engineering is a well-developed approach for designing and managing complex engineering systems consisting of hardware, software, and people. Many defense and technology organizations have published guidebooks for the system engineering process, and there are also several standard reference texts (Sage and Armstrong 2000). In the approach proposed, a fairly simple implementation of the key concepts of system engineering is viewed as a sufficient starting point for the structural design problem. The key concepts are to:

Start with the top-level performance parameters of interest to the overall vessel design problem considering the entire lifecycle of the vessel. Identify those that are impacted by the structural subsystem of the vessel. These may be overall properties such as structural weight, annual maintenance cost, production cost, etc.

Decompose these overall performance parameters into a list of more specific technical performance measures related to the structure, broadly following the intent of the functional allocation/analysis phase of systems engineering.

Synthesize a structural design that meets the technical performance measures, and compute the corresponding top-level structural performance metrics. Validation approaches for both the technical and top-level metrics are determined and applied.

- Validation continues throughout the structure's life, and future maintenance needs are forecast and decided upon based upon the structural condition and forecasts of specific structural performance metrics.

A key advantage of such an approach is that it provides a safeguard against the often-false economy of assuming a lighter structure is a less expensive structure. Without such an approach, acceptable structural performance is often assumed during the design by asserting compliance with existing rule sets. As structural performance metrics are rarely included in the KPP and KSA required for the design, any assumptions or margin reductions in the structures category, which allows for a lower initial cost or weight appear to be advantageous to the overall program. Unfortunately, such optimistic thinking often leads to difficulties in realizing an acceptable structure in the detail design phase or inservice failures requiring lengthy repairs and down time.

Given the importance of structural integrity to the safety of life on the vessel, as well as to the vessel itself and the environment, it is suggested that a hybrid approach will be required in setting up such a structural system model. A minimum threshold level for safety-related KPP would be set by existing approaches to structural design, such as the Naval Vessel Rules. Beyond this, different design efforts could propose their own structure-related KPP that could be used to 
compare and optimize different structures. A hypothetical high-level breakdown of such KPP is given in Figure 3, following the high-level metrics proposed by Hess (2002) and showing a partial breakdown of a single high-level KPP for a shell plate structural component.

While there are clear advantages to a more systems-oriented view of the structural design problem, significant development is required before such an approach is practical. The first and most pressing of these is the development of structural analysis tools and approaches that can provide the lower-level structural subsystem performance data required to compute the toplevel KPP assigned to the structure. These methods must be able to predict, if stochastically, variations in the KPP with small changes in the material description of the structure so that the naval architect may optimize structures for the higher-level program objectives expressed through the KPP. Structural reliability theory is one potential approach to such a formulation, although in its current form it does not possess all the required attributes for this application
(Collette et al. 2005). As the structural community does not have a long tradition of producing such calculations in an absolute frame of reference, accuracy, or at least confidence intervals on the results of such calculations are critical. Once variations in the structural KPP are exposed as part of the overall vessel design problem, it is only natural that the naval architect will trade off resources invested in structure against resources invested in machinery, combat systems, or other subsystems in order to find the most effective manner of meeting the customer's requirements for the vessel's performance. Thus, figures in an absolute frame of reference are required for use in a systems engineering approach.

Additionally, certain aspects of the structural design problem also do not map easily into a systems-engineering approach. Much of systems engineering has been developed for software, mechanical, or similar systems composed of discrete elements with defined interactions. Unfortunately, the most common structural system breakdown-largely based on existing

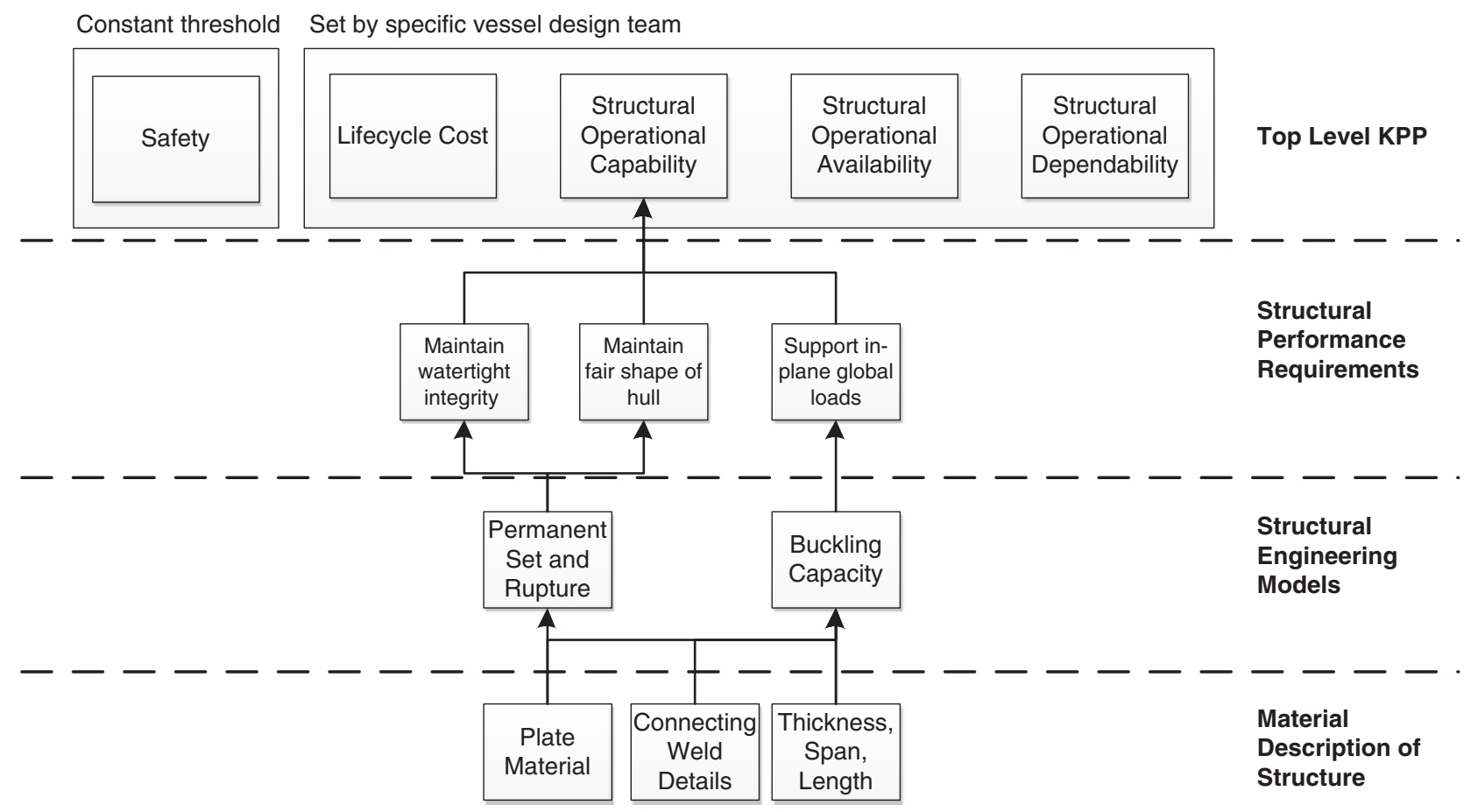

Figure 3: Hypothetical Breakdown in KPP, Showing Partial Breakdown for Plating Capability 
analysis tools-into plates, stiffened panels, grillages, compartments, and hulls, does not allow such clean boundaries to be drawn between systems. For example, a plate in the bottom shell is participating in the structural response at all levels in the previous breakdown list. This means that there is not a straightforward method of partitioning the structure and defining specific objectives for each member, as the performance of the components are highly interlinked. The optimum method for representing vessel structure in a systems engineering approach and handling the resulting interactions still requires exploration. Finally, much of the systems engineering work published to date has focused on the initial design of the system in question. While this is an important aspect, ongoing maintenance and repair is a large part of the operating cost of naval structures, and extensions of the system engineering approach into this aspect of the problem are also required.

\section{Comparative Example}

A simple example of the shortcomings of the current structural design approach and a potential reformulation of the problem in a systemoriented structural design approach is presented in the area of structural fatigue cracking. Structural fatigue cracking of naval structures is a major structural design concern for both new vessels and vessels in service. Currently, fatigue life is primarily assessed by the $\mathrm{S}-\mathrm{N}$ fatigue approach combined with the Palmgren-Miner cumulative damage rule, leading to an expression for the number of applied loading cycles until a crack occurs, $N_{\mathrm{I}}$, as:

$$
N_{\mathrm{I}}=\frac{D_{\mathrm{cr}} A}{\Delta \sigma^{m}}
$$

where $A$ and $m$ are experimentally determined constants, $\Delta \sigma$ is the equivalent stress range acting on the fatigue location, and $D_{\mathrm{cr}}$ is the cumulative damage index from the PalmgrenMiner cumulative damage rule. $A, m$, and $D_{\text {cr }}$ are empirically based, and the coefficients are typically determined by a fatigue test program. It is normal for there to be a large amount of experimental scatter in such fatigue test programs, with otherwise-identical specimens often failing at fatigue lives that differ by an order of magnitude. To handle this statistical scatter, a design value of the coefficients $A, m$, and $D_{\mathrm{cr}}$ are usually chosen, often two or more standard deviations off the mean and used to approve the details on the structure.

While this approach has resulted in the design of structures with a generally acceptable level of fatigue performance, it is not directly suitable for a systems-based approach. Fatigue cracking is a major LCC driver; however, the existing approach does not predict the actual number of cracks that are likely to be encountered over the vessel's service life. If the critical fatigue detail under consideration is a longitudinal-frame intersection, then under the existing approach, as the longitudinal or frame spacing is varied, the number of details in the vessel will change, as will the number of potential cracks in the vessel's service life. However, the current design approach does not allow this effect to be assessed, it simply indicates if a single detail is acceptable or not in isolation. Additionally, the probability of a crack occurring at any location has been fixed when the fatigue design coefficients are selected, however, in a systems view this probability may be further reduced by the design team-it may be more cost-effective from a lifecycle point of view to reduce the probability of fatigue cracking so that the through-life repair cost can be reduced. To go beyond the existing approach, it is necessary to expose the probabilistic fatigue model to the vessel design team. For this example, a probabilistic interpretation of equation (1) is taken, with both $A$ and $D_{\text {cr }}$ following lognormal distributions, and for simplicity, no uncertainty is associated with the loading stress. The lognormal distribution has the following probability density function, and has been shown previously to be a reasonable fit for ship-like structure fatigue data (Collette and Incecik 2006):

$$
p(x)=\frac{1}{\zeta \sqrt{2 \pi} x} \exp \left(-\frac{(\ln (x)-\lambda)^{2}}{2 \zeta^{2}}\right)
$$


The lognormal distribution is well suited for this type of modeling, as it is only defined for positive values, and the shape of the distribution is generally well suited for the type of scatter seen in fatigue tests. In equation (2) the parameters of the lognormal distribution are $\zeta$ and $\lambda$. Under the assumption that the stochastic variables in equation (1) follow the lognormal distribution, the distribution of $N_{\text {I }}$ will also follow a lognormal distribution with the following parameters:

$$
\begin{aligned}
& \lambda_{I}=\lambda_{D_{\mathrm{cr}}+\lambda_{A}-m(\ln (\Delta \sigma))} \\
& \zeta_{I}=\sqrt{\zeta_{D_{\mathrm{cr}}}^{2}+\zeta_{A}^{2}}
\end{aligned}
$$

With a further assumption that each as-built fatigue detail is an independent realization of the distribution from equation (3), it is then possible to determine the number of fatigue cracks expected to initiate in each year of the structure's life. The results of such as calculation are shown below for a nominal $150 \mathrm{~m}$ long combatant. In this example, the highest-stressed critical longitudinal/web frame intersect fatigue details in the strength deck are designed for $97.5 \%$ probability of survival for a 30-year service life using current design approaches. The annual number of cracks expected is calculated under two different structural designs-one with a $1.5 \mathrm{~m}$ web frame spacing $(F)$, and a $400 \mathrm{~mm}$ longitudinal spacing $(s)$, and one with a $2 \mathrm{~m}$ web frame spacing and $500 \mathrm{~mm}$ longitudinal spacing. It is assumed that the longitudinal bending stresses are the dominant fatigue loads, and are identical in each structure. The midship $60 \mathrm{~m}$ of the strength deck is analyzed, with the bending moment varying with a $1-\cos ^{2}$ distribution along the length of the hull. The results are shown in Figure 4; the structure with more fatigue-critical details produces more cracks in service. This is not a surprising conclusion; however, current structural design philosophy would treat both these structures as identical as the rate of fatigue crack initiation is the same in each structure at each detail. It is not until a system view of the structure is taken that the need for different fatigue calculations becomes apparent. However, the proposed probabilistic approach does provide the overall design team feedback on the number of likely cracks and hence repairs required in service, which can then be used to compute structural maintenance and capability metrics at higher levels.

\section{Conclusions}

The case for taking a systems-oriented view to structural design is presented. Existing structural design processes are reviewed, and are shown to primarily use empirically linked load definitions, analysis tools, and acceptance criteria. As these approaches do not directly address the actual failures, which may occur in a structure, it is difficult to use the results of such analysis when attempting to predict the lifecycle capability and cost performance of the structure. Extensions beyond the existing approach have been proposed, such as GBS to move toward a more

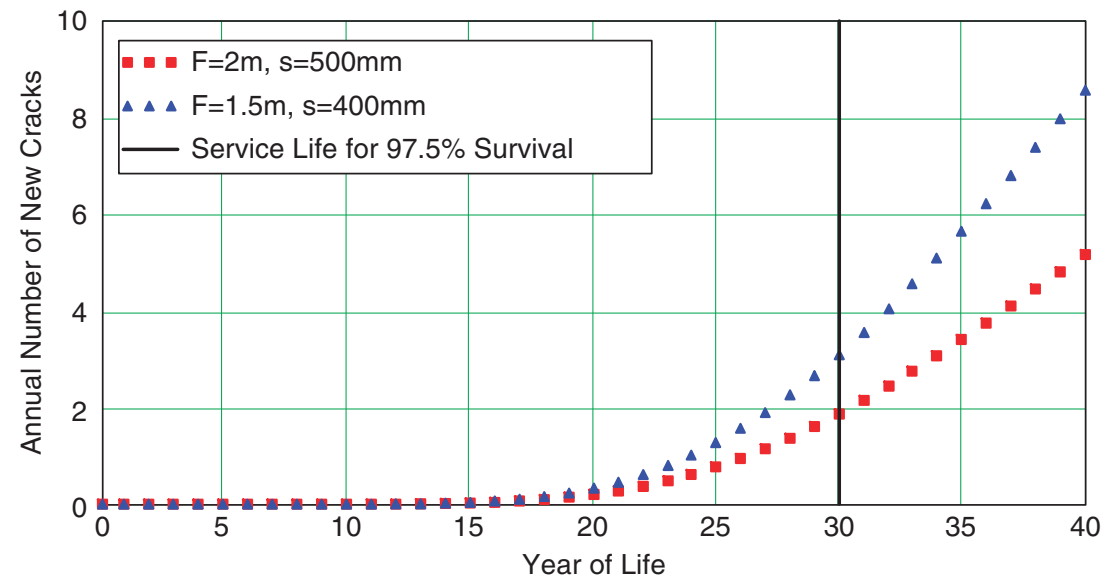

Figure 4: Annual Crack Occurrence with Different Number of Fatigue Details 
consistent level of safety in the structure, or design-for-production approaches which generate metrics for the producability of the structure. It is argued that these approaches are essentially ad hoc extensions beyond the existing stateof-the-art. However, such approaches can be joined together into a logical, prioritized framework by taking a systems view of the structure and its impact on the overall capabilities and cost of the vessel design. Potential difficulties in making this transition are discussed; primarily they are seen to be structural tools capable of producing the required metrics with associated measures of uncertainty critical for combining structural metrics with other design metrics in an absolute frame such as cost effectiveness. Additionally, difficulties in following traditional system engineering partitioning and decomposition approaches with structural systems are seen. A simple example of different structural design approaches for fatigue cracking has been used to illustrate the shortcomings of the current calculation tools and give an example of the type of approach that could be taken in a more systemsoriented view of the structure.

\section{Hcknowledgments}

The author wishes to acknowledge the work Mark Groden of the University of Michigan performed for compiling the service lives of numerous US Navy vessels and tracking down many of the key documents on GBS. The support of Dr. Paul Hess of Code 331 of ONR on probabilistic fatigue analysis is also gratefully acknowledged, as well as his helpful discussions on the philosophy of structural design and analysis for warships.

\section{References}

Barentine, J., "A process-based cost estimating tool for ship structural designs." Available at http://dspace. mit.edu/handle/1721.1/10557. Accessed October 22, 2010.

Collette, M., M. Cooper, A. Mesbahi, and A. Incecik, "An integrated reliability, risk analysis, and cost model for preliminary structural design: a module of the SAFETY@SPEED design methodology," In FAST 2005. Eighth International Conference on Fast Sea Transportation. St. Petersburg, Russia, 27-30 June 2005.
Collette, M. and A. Incecik, "An approach for reliabilitybased fatigue design of welded joints on aluminunn high-speed vessels," Journal of Ship Research, Vol. 50, No. 1, pp. 85-98, 2006.

Hess, P., "Reliability-based operational performance metrics for ship structures." Ph.D. thesis, University of Maryland, 2002.

Hoppe, H., "Goal-based standards - a new approach to the international regulation of ship construction," WMU Journal of Maritime Affairs, Vol. 4, No. 2, pp. 169-180, 2005.

Koenig,, P., D. Nalchajian, and J. Hootman, "Ship service life and naval force structure," Naval Engineers Journal, Vol. 121, No. 1, pp. 69-77, 2009.

NATO, "ANEP 77 Naval Ship Code." North Atlantic Treaty Orģanization, July 2010.

OPNAV, "Operational Availability Handbook." Department of the Navy, Office of the Chief of Naval Operations Instruction, 1987.

Rigo, P., "Least-cost structural optimization oriented preliminary design," Journal of Ship Production, Vol. 17, pp. 202-215, 2001.

Saģe, A.P. and J.E. Armstrong Jr., Introduction to systems enģineering, 1st ed., Wiley-Interscience, New York, 2000.

Sames, P.C. and R. Hamann, "Towards environmental risk acceptance criteria," In 27th International Conference on Offshore Mechanics and Arctic Enogineering, OMAE 2008, June 9, 2008-June 13, 2008, Estoril, Portugal. ASME Vol. 2, pp. 277-283, 2008.

Sielski, R., SSC-452 Aluminum structure design and fabrication guide, Ship Structure Committee, Washinģton, DC, 2007.

Turan, O., A.İ. Ölçer, I. Lazakis, P. Rigo, and J.D. Caprace, "Maintenance/repair and production-oriented life cycle cost/earning model for ship structural optimisation during conceptual design stage," Ships and Offshore Structures, Vol. 4, No. 2, pp. 107-125, 2009.

\section{Huthor Biography}

Dr. Matthew Collette joined the Naval Architecture and Marine Engineering Department of the University of Michigan in 2009 as an Assistant Professor. His research focuses on the application of numerical methods to design and 
operational support, with a focus on lifecycle structural response including lightweight structures, structural optimization, and stochastic methods. He is currently an associate editor for Ocean Engineering. Before joining Michigan, he worked at SAIC as a Senior Naval Architect supporting a variety of governmental and commercial research projects covering hydrodynamic analysis, optimization, and computational technologies for design and operation, and analysis tools for lightweight structures. Dr. Collette started his career in Boston working for John W. Gilbert Associates as a naval architect. He is a 1999 graduate of Webb Institute and completed his PhD at the University of Newcastle in the United Kingdom in 2005. Currently at University of Michigan, Department of Naval Architecture and Marine Engineering, Room 210, 2600 Draper Drive, Ann Arbor, MI 48109, 734-764-8422.

E-mail:mdcoll@umich.edu 